
JNM
J Neurogastroenterol Motil, Vol. 22 No. 2 April, 2016
pISSN: 2093-0879 elSSN: 2093-0887
http://dx.doi.org/10.5056/jnm15132

\title{
High-resolution Manometry Findings in Patients After Sclerotherapy for Esophageal Varices
}

\author{
Fernando A M Herbella, ${ }^{1 *}$ Ramiro Colleoni, ${ }^{1}$ Luiz Bot, ${ }^{1}$ Fernando P P Vicentine, ${ }^{1}$ and Marco G Patti ${ }^{2}$ \\ ${ }^{I}$ Department of Surgery, Escola Paulista de Medicina, Federal University of Sao Paulo, São Paulo, Brazil; and ${ }^{2}$ Department of Surgery, University \\ of Chicago, Chicago, USA
}

\section{Background/Aims}

Endoscopic therapy for esophageal varices may lead to esophageal dysmotility. High-resolution manometry is probably the more adequate tool to measure esophageal motility in these patients. This study aimed to evaluate esophageal motility using high resolution manometry following eradication of esophageal varices by endoscopic sclerotherapy.

\section{Methods}

We studied 21 patients (11 women, age 52 [45-59] years). All patients underwent eradication of esophageal varices with endoscopic sclerotherapy and subsequent high resolution manometry.

\section{Results}

A significant percentage of defective lower esophageal sphincter (basal pressure 14.3 [8.0-20.0] mmHg; $43 \%$ hypertonic) and hypocontractility (distal esophageal amplitude 50 [31-64] mmHg; proximal esophageal amplitude 40 [31-61] mmHg; distal contractile integral 617 [403-920] $\mathrm{mmHg} \cdot \mathrm{sec} \cdot \mathrm{cm} ; 48 \%$ ineffective) was noticed. Lower sphincter basal pressure and esophageal amplitude correlated inversely with the number of sessions $(P<0.001)$. No manometric parameter correlated with symptoms or interval between last endoscopy and manometry.

\section{Conclusions}

Esophageal motility after endoscopic sclerotherapy is characterized by: (1) defective lower sphincter and (2) defective and hypotensive peristalsis. Esophageal dysmotility is associated to an increased number of endoscopic sessions, but manometric parameters do not predict symptoms.

(J Neurogastroenterol Motil 2016;22:226-230)

Key Words

Esophageal motility disorders; Esophageal varices; Hypertension, portal; Manometry; Sclerotherapy

\section{Introduction}

Esophageal varices are secondary to portal hypertension due to the presence of a peri-esophageal portosystemic collateral circula- tion. ${ }^{1}$ Although liver transplantation and trans-jugular intrahepatic portosystemic stent-shunts (TIPSS) are established methods to treat portal hypertension and consequently esophageal varices, different forms of endoscopic therapy have long been used for variceal bleeding prophylaxis and therapy. ${ }^{2}$

Received: August 18, 2015 Revised: October 7, 2015 Accepted: November 2, 2015

(a) This is an Open Access article distributed under the terms of the Creative Commons Attribution Non-Commercial License (http://creativecommons. org/licenses/by-nc/4.0) which permits unrestricted non-commercial use, distribution, and reproduction in any medium, provided the original work is properly cited.

${ }^{*}$ Correspondence: Fernando A M Herbella, MD Department of Surgery, Escola Paulista de Medicina, Rua Diogo de Faria 1087 cj 301 Sao Paulo, SP, Brazil 04037-003 Tel: +55-11-99922824, Fax: +55-11-39267610, E-mail: herbella.dcir@epm.br 
Endoscopic therapy for esophageal varices may lead to esophageal dysmotility due to the process of scarring. Previous reports focused on the alteration of esophageal motility after endoscopic therapy. ${ }^{3-13}$ These studies, however, relied on conventional manometry for the evaluation. High-resolution manometry (HRM) is probably a more adequate tool to measure esophageal motility in these patients. HRM allows a sophisticated evaluation of the lower esophageal sphincter (LES) relaxation and of segmental peristaltic defects since altered peristalsis may occur only at the injection point. $^{14}$

This study aimed to evaluate esophageal motility using HRM following eradication of esophageal varices by endoscopic sclerotherapy.

\section{Materials and Methods}

We studied 21 consecutive patients who had undergone esophageal variceal eradication by endoscopic sclerotherapy in the past and volunteered for the prospective evaluation of esophageal function (11 women, mean age 52 [45-59] years).

Patients with previous foregut surgery, moderate or severe ascites, primary esophageal motility disorders, or systemic diseases that affect esophageal motility were not recruited to the study.

\section{Symptoms}

Patients were questioned about dysphagia and esophageal symptoms of gastroesophageal reflux disease at the time of the HRM. Symptoms were considered positive if dysphagia occurred at least weakly for any type of food and heartburn or regurgitation occurred at least weekly.

\section{Endoscopic Therapy}

All patients had variceal bleeding in the past and had medium of large size varices. Monthly endoscopic intraluminal injection of $2 \%$ ethanolamine oleate was performed in all patients until eradication. The sclerosant volume injected varied from $3 \mathrm{~mL}$ to $18 \mathrm{~mL}$ with a median of $9 \mathrm{~mL}$, and decreasing doses for progressive sessions. ${ }^{15}$

The median number of sessions was 6 (4-10). The interval between the final sclerotherapy session and the esophageal manometry was 25 (12-94) months.

\section{High-resolution Manometry}

HRM was performed as previously described. ${ }^{16}$ In summary, patients fasted for at least 8 hours and discontinued any medications that interfered with esophageal and gastric motility 3 days before the study. A solid-state catheter with 36 circumferential sensors spaced $1 \mathrm{~cm}$ was used (Medtronics, Los Angeles, CA, USA). The test was performed and analyzed according to the manufacturer's instructions and dedicated software. Ten wet swallows of $5 \mathrm{~mL}$ water boluses at 30 -second intervals were offered to allow the recording of: (1) the position, pressure (defined as the mid-expiratory pressure), relaxation (as defined by the integrated relaxation pressure), and length of the LES, (2) amplitude, duration and propagation of the peristaltic waves at $3 \mathrm{~cm}$ and $7 \mathrm{~cm}$ above the LES, and (3) segmental defects of peristalsis based on visual analysis.

The normal values considered in this study derived from the Chicago group per system manufacturer software. They were LES length $>2.7 \mathrm{~cm}$, LES basal pressure 13-43 $\mathrm{mmHg}$, LES residual pressure $<15 \mathrm{mmHg}$, distal esophageal amplitude (DEA; sensor located $3 \mathrm{~cm}$ above the upper border of the LES) $41-168 \mathrm{mmHg}$, and proximal esophageal amplitude (sensor located $7 \mathrm{~cm}$ above the upper border of the LES) 37-166 mmHg. Distal contractile integral (DCI) defined esophageal contractions as ineffective (failed + weak) if $<800 \mathrm{mmHg} \cdot \mathrm{sec}^{\cdot} \mathrm{cm}$ or hypercontractile if $>8000$ $\mathrm{mmHg} \cdot \mathrm{sec} \cdot \mathrm{cm}$ and distal latency $(\mathrm{sec})<4.5$ seconds defined a premature contraction.

\section{Statistical Methods}

Variables are expressed as median (interquartile range), as a non-normal distribution of the data was found by the Shapiro-Wilk test. Spearman correlation or Mann-Whitney tests were used when appropriated. A value of $P$ was considered significant at the 0.05 level.

\section{Ethics}

The study was approved by the Institutional Review Board. There are no conflicts of interest. The authors are responsible for the manuscript and no professional or ghost writers were hired.

\section{Results}

HRM was feasible in all patients without any complications. Dysphagia was reported by 8 (38\%) patients, and reflux symptoms were reported by $10(48 \%)$ patients.

\section{Lower Esophageal Sphincter}

Manometric parameters for the LES are shown in our Table. A significant percentage of patients had a defective LES. LES lengths (total and abdominal) did not correlate with symptom pres- 
ence, number of sclerotherapy sessions, or interval between the manometry and the final session of sclerotherapy. LES basal pressure correlated inversely with the number of sessions $(P<0.001)$ (Fig. 1), but did not correlate with dysphagia $(P=0.700)$, reflux symptoms $(P=0.500)$ or interval between the final session and the $\operatorname{HRM}(P=0.100)$. LES relaxation did not correlate with dysphagia $(P=0.600)$, reflux symptoms $(P=0.700)$, number of sessions $(P=0.200)$, or interval between the final session and the HRM $(P$ $=0.300)$.

\section{Esophageal Body}

Manometric parameters for the esophageal body are shown in our table. DEA correlated with the number of sessions $(P<0.001)$ (Fig. 1) but not with symptoms of dysphagia $(P>0.99)$ or reflux $(P$ $=0.600)$ or interval between the final session and the $\operatorname{HRM}(P=$ 0.400). Proximal esophageal amplitude showed similar results with a correlation with the number of sessions $(P=0.010)$ (Fig. 1) but not with symptoms $(P>0.99)$ and time from last endoscopic treatment $(P=0.400)$. DCI defined ineffective peristalsis in almost half of the patients. DCI did not correlate with dysphagia $(P>0.99)$, reflux symptoms $(P>0.99)$, the number of sessions $(P=0.400)$,

Table. Manometric Findings in Patients After Endoscopic Sclerotherapy $(\mathrm{n}=21)$

\begin{tabular}{lc}
\hline LES basal pressure (median [IQR], mmHg) & $14.3(8.0-20.0)$ \\
Hypotonic & $43 \%$ \\
Hypertonic & 0 \\
LES residual pressure (median [IQR], mmHg) & $4.8(1.6-7.2)$ \\
Abnormal relaxation & $5 \%$ \\
LES length (median [IQR], cm) & $2.6(2.3-3.2)$ \\
Short & $95 \%$ \\
LES abdominal length (median $[\mathrm{IQR}], \mathrm{cm})$ & $1.7(1.0-2.4)$ \\
Distal esophageal amplitude & $50(31-64)$ \\
(median [IQR], mmHg) & 0 \\
Hypercontractility & $33 \%$ \\
Hypocontractility & \\
Proximal esophageal amplitude & $40(31-61)$ \\
(median [IQR], mmHg) & 0 \\
Hypercontractility & $38 \%$ \\
Hypocontractility & \\
Distal contractile integral & $617(403-920)$ \\
(median [IQR], mmHg·sec·cm) & $48 \%$ \\
Ineffective & 0 \\
Hypercontractility & $5.8(4.6-7.2)$ \\
Distal latency (median $[\mathrm{IQR}], \mathrm{sec})$ & $24 \%$ \\
Premature & \\
\hline
\end{tabular}

LES, lower esophageal sphincter; IQR, interquartile range. and time from last endoscopic treatment $(P=0.900)$. Distal latency did not correlate with dysphagia $(P=0.600)$, reflux symptoms $(P$ $=0.600)$, the number of sessions $(P=0.500)$, and time from last endoscopic treatment $(P=0.600)$. Two $(9 \%)$ patients had hypercontractile segments (Fig. 2). No patient presented with pathologic peristaltic gaps.

\section{Discussion}

There is no clinical reason to suppose that portal hypertension per se or for that matter cirrhosis or schistosomiasis, 2 important causes of portal hypertension - lead to alteration in esophageal mo-

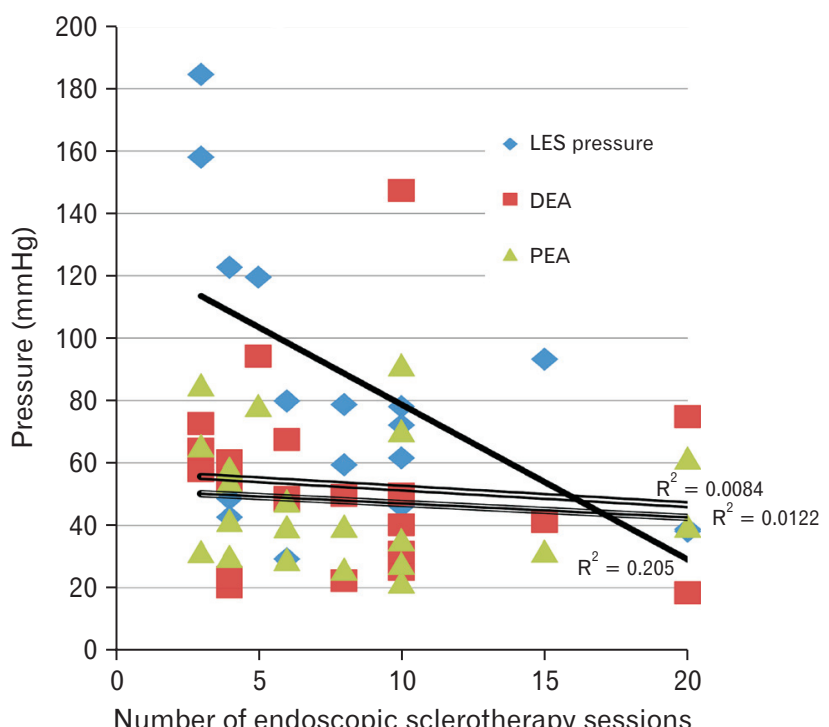

Figure 1. Correlation between number of endoscopic sclerotherapy sessions and lower esophageal sphincter (LES) basal pressure, distal esophageal amplitude (DEA), and proximal esophageal amplitude (PEA).

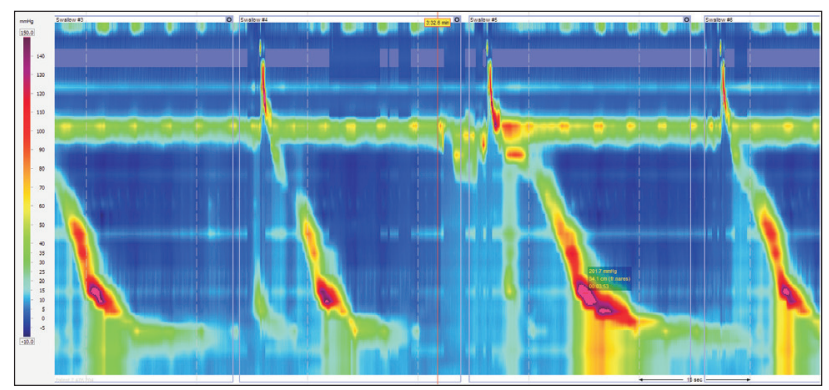

Figure 2. Example of hypercontractile distal segments after endoscopic sclerotherapy for esophageal varices with a distal contractile integral $=2050 \mathrm{mmHg} \cdot \mathrm{sec} \cdot \mathrm{cm}$. 
tility unless a systemic disease is the etiology of the liver failure. ${ }^{17} \mathrm{In}$ fact, there are no studies that have focused exclusively on the manometric testing of these patients. Secondary manifestations of portal hypertension may, however, influence esophageal motility. The presence of ascites does not alter LES pressure ${ }^{18,19}$ but the presence of varices has been associated with decreased DEA for some ${ }^{20}$ although questioned by others. ${ }^{10}$

Endoscopic therapy for esophageal varices may affect esophageal motility due to the development of esophageal fibrosis. Previous studies showed motility changes after injection sclerotherapy, ${ }^{3,9,10}$ while others only noticed manometric changes if esophageal stenosis $^{4}$ or dysphagia ${ }^{5}$ were present, or acutely after 24 hours following the injection. ${ }^{7}$ Studies on rubber band variceal ligation found no changes in esophageal motility compared to patients before endoscopic therapy or sclerotherapy ${ }^{21}$ apart from transitory increased body amplitude. ${ }^{8}$

The LES is at risk of fibrosis after endoscopic sclerotherapy since injections are usually performed close to the esophagogastric junction. Conventional manometry series are contradictory with regard to LES basal pressure after endoscopic therapy for varices. Some authors noticed a decrease in pressure ${ }^{3,10,13}$ while others did not show any changes. ${ }^{5-7}$ Our results showed a significant percentage of patients with hypotonic LES and a positive correlation between the number of sclerotherapy sessions and LES basal pressure. In fact, Sharma et $\mathrm{al}^{13}$ showed not only the same results but also noticed that the degree of chronic inflammatory cell infiltrate and fibrosis parallels the number of sessions. There is no previous data on LES relaxation. We hypothesized that LES fibrosis would also impair its relaxation; however, our results did not support this idea even with the use of sophisticated analysis with the HRM parameter the integrated relaxation pressure. ${ }^{22}$

Even though sclerosing agents are injected into the distal esophagus only, the centrifuge blood flow may cause the agent to ascend and contribute to fibrosis of the proximal esophagus as well. Lower $\mathrm{DEA}^{3}$ and higher percentage of non-peristaltic waves ${ }^{3,5}$ has been reported after sclerotherapy in conventional manometry studies. HRM also showed a significant proportion of defective and hypotensive peristalsis, directly proportional to the number of endoscopic sessions. Hypercontractile segments on the distal esophagus were noticed in almost $10 \%$ of our patients. These segmental abnormalities were missed on the automated analysis and they would be, obviously, not diagnosed by conventional manometry.

Manometric parameters did not correlate with symptoms except for dysphagia that occured more frequently in patients with a higher LES pressure irrespective of its relaxation.
The current study has some limitations. First, it included a low number of patients. Although the number of studied patients matches other similar studies, it is small since the esophageal function tests are not part of their care and they volunteered to the study irrespective of symptoms. Second, esophageal manometry was not performed before the beginning of the endoscopic sessions to allow a comparison as a control group. Although HRM was done prospectively, patients were recruited after retrospective endoscopic therapy. Thus, the duration between the final therapy and the HRM was not uniform. The strength of the study is the long follow up and the application of sophisticated methodology with HRM.

In conclusion, our results showed that esophageal motility after endoscopic sclerotherapy for esophageal varices is characterized by: (1) defective LES and (2) defective and hypotensive peristalsis. Esophageal dysmotility is associated to an increased number of endoscopic sessions, but manometric parameters do not predict symptoms. The number of endoscopic sclerotherapy sessions must be minimized or switched to a more efficient method, such as rubber band ligation.

Acknowlegements: We are indebted to Ms. Vanessa Horich Tuxen for her invaluable assistance with the esophageal tests.

Financial support: None.

Conflicts of interest: None.

Author contributions: Fernando A M Herbella: conception and design, acquisition of data, analysis and interpretation of data, drafting the article, and final approval of the version to be published; Ramiro Colleoni: conception and design, acquisition of data, analysis and interpretation of data, and final approval of the version to be published; Luiz Bot and Fernando P P Vicentine: acquisition of data, analysis and interpretation of data, and final approval of the version to be published; and Marco G Patti: review for intellectual content and final approval of the version to be published.

\section{References}

1. Iwakiri Y. Pathophysiology of portal hypertension. Clin Liver Dis 2014;18:281-291.

2. Rajoriya N, Tripathi D. Historical overview and review of current day treatment in the management of acute variceal haemorrhage. World J Gastroenterol 2014;20:6481-6494.

3. Sauerbruch T, Wirsching R, Leisner B, Weinzierl M, Pfahler M, 
Paumgartner G. Esophageal function after sclerotherapy of bleeding varices. Scand J Gastroenterol 1982;17:745-751.

4. Cohen LB, Simon C, Korsten MA, et al. Esophageal motility and symptoms after endoscopic injection sclerotherapy. Dig Dis Sci 1985;30:29-32.

5. Söderlund C, Thor K, Wiechel KL. Oesophageal motility after sclerotherapy for bleeding varices. Acta Chir Scand 1985;151:249-253.

6. Bovero E, Farese A. Manometric evaluation of esophageal motility in patients submitted to prophylactic variceal sclerosis. Surg Endosc 1988;2:156-158

7. Grande L, Planas R, Lacima G, et al. Sequential esophageal motility studies after endoscopic injection sclerotherapy: a prospective investigation. Am J Gastroenterol 1991;86:36-40.

8. Kim HC, Song JH, Kim HE, et al. Effects of endoscopic variceal ligation in lower esophageal motor function: a prospective study. Korean J Intern Med 1995;10:120-124.

9. Isaksson B, Johnsson F, Jeppsson B. Long-term effects of repeated injection sclerotherapy on esophageal motility and mucosa. Surg Endosc 1997;11:663-667.

10. Ghoshal UC, Saraswat VA, Aggarwal R, Misra A, Dhiman RK, Naik SR. Oesophageal motility and gastro-oesophageal reflux: effect of variceal eradication by endoscopic sclerotherapy. J Gastroenterol Hepatol 1998;13:1033-1038.

11. Narawane NM1, Bhatia SJ, Sheth MD, et al. Early changes in esophageal motility after endoscopic variceal sclerotherapy or ligation. Indian J Gastroenterol 1999;18:11-14.

12. Ghoshal UC, Dhar K, Chaudhuri S, Pal BB, Pal AK, Banerjee PK. Esophageal motility changes after endoscopic intravariceal sclerotherapy with absolute alcohol. Dis Esophagus 2000;13:148-151.

13. Sharma P, Hagerstrand I, Sharma DK. Histologic and manometric studies on the esophagus following endoscopic sclerotherapy. Dig Dis Sci 2009;54:1713-1719.

14. Herbella FA, Patti MG. Can high resolution manometry parameters for achalasia be obtained by conventional manometry? World J Gastrointest Pathophysiol 2015;6:58-61.

15. Technology Assessment Committee, Croffie J, Somogyi L, et al. Sclerosing agents for use in GI endoscopy. Gastrointest Endosc 2007;66:1-6.

16. Martinelo V, Herbella FA, Patti MG. High-resolution manometry findings in patients with an intrathoracic stomach. Am Surg 2015;81:354357.

17. Bektas M, Seven G, Idilman R, et al. Manometric assessment of esophageal motor function in patients with primary biliary cirrhosis. Eur J Intern Med 2014;25:230-234.

18. Eckardt VF, Grace ND, Kantrowitz PA. Does lower esophageal sphincter incompetency contribute to esophageal bleeding? Gastroenterology 1976;71:185-189.

19. Bhatia SJ, Narawane NM, Shalia KK, et al. Effect of tense ascites on esophageal body motility and lower esophageal sphincter pressure. Indian J Gastroenterol 1999;18:63-65.

20. Passaretti S, Mazzotti G, de Franchis R, Cipolla M, Testoni PA, Tittobello A. Esophageal motility in cirrhotics with and without esophageal varices. Scand J Gastroenterol 1989;24:334-348.

21. Goff JS, Reveille RM, Van Stiegmann G. Endoscopic sclerotherapy versus endoscopic variceal ligation: esophageal symptoms, complications, and motility. Am J Gastroenterol 1988;83:1240-1244.

22. Kahrilas PJ, Bredenoord AJ, Fox M, et al. The Chicago classification of esophageal motility disorders, v3.0. Neurogastroenterol Motil 2015;27:160-174. 